\title{
ARTICLE \\ CS1003, a novel human and mouse cross-reactive PD-1 monoclonal antibody for cancer therapy
}

Fu Li ${ }^{1}$, Jingrong $\mathrm{Li}^{1}$, Ke Yin ${ }^{1}$, Juan Zhang ${ }^{1}$, Zhen-hu Li ${ }^{1}$, Liang $\mathrm{Lu}^{1}$, Yuan-wu Bao ${ }^{1}$, Zhen Qin ${ }^{1}$, Yong Zheng ${ }^{2}$, Bao-tian Yang ${ }^{2}$, Jing $\mathrm{Li}^{2}$ and Xinzhong Wang ${ }^{1}$

The programmed cell death protein 1 (PD-1) is an immune-checkpoint that negatively regulates the immune system and a key mechanism that tumors utilize to escape from immune surveillance. PD-1 antibodies can block the interaction of PD-1 with its ligands (PD-L1 and PD-L2), restore T cells activation, and elicit antitumor activity. In this paper, we reported a novel PD-1 monoclonal antibody (mAb) CS1003, which is a humanized lgG4 PD-1 mAb generated by conventional hybridoma technology, and currently being developed in multiple clinical trials as monotherapy or in combination with other anticancer agents. We showed that CS1003 bound to recombinant human, cynomolgus monkey, and mouse PD- 1 with EC $_{50}$ values of $0.1757,0.2459$, and 0.3664 $\mathrm{nM}$, respectively. CS1003 blocked PD-1 interaction with its ligands, dose-dependently enhanced T cell proliferation and secretion of cytokines (IL-2 and IFN- $\gamma$ ) to the levels comparable to the reference antibody pembrolizumab. Intraperitoneal administration of CS1003 $(0.1,0.5,2.5 \mathrm{mg} / \mathrm{kg}$, once every 3 days) dose-dependently suppressed the growth of MC38-hPD-L1 colon cancer in hPD-1 knock-in mice. Pharmacokinetics (PK) study revealed a linear PK profile within the dose range of 2-18 mg/kg following single intravenous administration in cynomolgus monkey. These data provide a comprehensive preclinical characterization of CS1003 that supports its clinical development for cancer immunotherapy.

Keywords: CS1003; monoclonal antibody; programmed cell death protein 1; programmed death-ligand 1; cancer immunotherapy

Acta Pharmacologica Sinica (2021) 42:142-148; https://doi.org/10.1038/s41401-020-0422-6

\section{INTRODUCTION}

The tumor immune microenvironment encompasses a wide range of complex interactions between tumor cells, immune cells, including DCs, T cells, NK cells, and B cells, and the tumor stroma [1]. Effective antitumor immune responses are the result of competition between inhibitory and stimulatory signals that regulate balanced immune responses in normal tissue. Immune checkpoints are important immune regulators in maintaining immune homeostasis and preventing autoimmunity. PD-1, a protein expressed on the surface of cells, plays an important role in downregulating immune responses and promoting selftolerance by suppressing $T$ cell inflammatory activity $[2,3]$. PD-1 was discovered and named by Tasuku Honjo and colleagues at Kyoto University in 1992 [4, 5]. To date, six anti-PD-1 antibodies have been approved for more than ten cancer indications by the FDA or NMPA. Among these, nivolumab was the first approved therapeutic anti-PD-1 monoclonal antibody (mAb) showing significant clinical efficacy in unresectable or metastatic melanoma with limited toxicity [6]. Pembrolizumab also showed significant antitumor efficacy and a good safety profile [7]. Although PD-1 mAbs demonstrate tremendous clinical success rates across multiple cancer types, $30 \%-60 \%$ of patients show no response to PD-1 blockade [8]. Currently, combination therapy is a key strategy to overcome the limitations of PD-1 monotherapy [9]. To evaluate the combined effect of anti-PD-1 mAbs in syngeneic mouse models with other agents, a surrogate antibody that recognizes mouse PD-1 such as RMP1-14 is often used in place of the actual therapeutic antibody. To our knowledge, none of the approved PD-1-targeting drugs recognizes mouse PD-1, and surrogate antibodies have been used for the evaluation of combination effects in syngeneic models. However, the efficacy of surrogate antibodies may not represent the actual effect in humans due to different binding epitopes.

In this paper, we report a novel human and mouse cross-reactive anti-PD-1 antibody, CS1003. It is a humanized IgG4 anti-PD-1 mAb generated by conventional hybridoma technology and developed for cancer immunotherapy. It shows comparable binding affinity, in vitro immune modulation and in vivo potency with reference antibodies. Our results suggest that $\mathrm{CS} 1003$ is not only an ideal therapeutic anti-PD-1 antibody candidate for cancer treatment but also a valuable tool for quickly testing combination therapy agents in syngeneic tumor models. A phase 1 study [NCT03475251] evaluating the safety, tolerability and pharmacokinetics (PK) properties of CS1003 in patients with advanced solid tumors is ongoing.

\section{MATERIALS AND METHODS}

Reagents

The anti-human PD-1 reference antibody pembrolizumab was purchased from Merck (Rahway, NJ, USA). CS1003, a humanized, hinge stabilized IgG4 mAb, nivolumab, and a human IgG4 isotype control antibody were synthesized by Wuxi Biologics (Shanghai,

${ }^{1}$ CStone Pharmaceuticals (Suzhou) Co., Ltd, Shanghai 201203, China and ${ }^{2}$ Wuxi Biologics Co. Ltd, Wuxi 214092, China

Correspondence: Xinzhong Wang (wangxinzhong@cstonepharma.com)

Received: 17 December 2019 Accepted: 17 April 2020

Published online: 28 May 2020 
Shanghai, China). Antigens from human PD-1, PD-L1, CTLA-4, CD28 and ICOS and mouse PD-1 and PD-L1 were prepared by Wuxi Biologics (Shanghai, China). A human IFN- $\gamma$ capture antibody and human IFN- $\gamma$ detection antibody were purchased from Pierce Biotechnology (Rockford, IL, USA).

SPR assay

The CM5 sensor chip was activated with EDC and NHS immediately before use. An antihuman Fc IgG antibody in $10 \mathrm{mM} \mathrm{NaAc}(\mathrm{pH} 4.5)$ was subsequently injected into Fc1-Fc4 for $420 \mathrm{~s}$ at a flow rate of $10 \mu \mathrm{L} / \mathrm{min}$. The chip was deactivated by $1 \mathrm{M}$ ethanolamine $\mathrm{HCl}$. Antibodies were diluted with running buffer $(1 \times$ HBS-EP + ) to $5 \mu \mathrm{g} / \mathrm{mL}$ and then captured onto a chip. Seven concentrations $(50,25,12.5,6.25,3.125,1.5625$, and $0.78125 \mathrm{nM})$ of human PD-1 (Sino Biological) and running buffer were injected into Fc1-Fc4. Glycine (10 mM, pH 1.5) used as regeneration buffer was injected following each dissociation phase.

\section{ELISA assay}

For the binding assay, plates were precoated with fusion protein at $4{ }^{\circ} \mathrm{C}$ overnight. After $1 \mathrm{~h}$ of blocking, testing antibodies were added to plates. The plates were incubated at room temperature for $1 \mathrm{~h}$. The binding of antibodies to immobilized proteins was detected by an HRP-labeled goat-anti-human IgG antibody. The signal was activated by dispensing the TMB substrate and then stopped by $\mathrm{HCl}$. The absorbance was measured at $450 \mathrm{~nm}$ and $540 \mathrm{~nm}$ using a microplate spectrophotometer.

\section{FACS assay}

Human PD-1-expressing CHO-S cells were incubated with serial dilutions of test anti-PD- 1 antibodies at $4{ }^{\circ} \mathrm{C}$ for $1 \mathrm{~h}$. Testing antibodies were serially diluted (1:2) in wash buffer $(1 \times \mathrm{PBS} / 1 \%$ BSA) starting from $16.67 \mathrm{nM}$. A FITC-labeled goat antihuman IgG was used to detect the binding of anti-PD-1 antibodies to the cells. The mean fluorescence intensity of cells was measured by a flow cytometer and analyzed by FlowJo.

Mixed lymphocyte reaction (MLR) assay

Human peripheral blood mononuclear cells (PBMCs) were freshly isolated from healthy donors using Ficoll-Paque PLUS gradient centrifugation. Isolated PBMCs were cultured in complete RPMI1640 medium (containing 10\% FBS and 1\% PS) supplemented with $100 \mathrm{U}$ recombinant human IL-2. $\mathrm{CD}^{+} \mathrm{T}$ cells were isolated from human PBMCs. Purified $\mathrm{CD}^{+}{ }^{+} \mathrm{T}$ cells were cocultured with immature or mature allogeneic DCs (iDCs or mDCs). The MLR assay was set up in 96-well round-bottom plates using complete RPMI1640 medium. $\mathrm{CD}^{+} \mathrm{T}$ cells, various concentrations of antibodies $(166.75,66.7,6.67,0.667,0.0667$, and $0.00667 \mathrm{nM})$, and allogeneic DCs were added to the plates at appropriate ratios. The plates were incubated at $37^{\circ} \mathrm{C}$ in $5 \% \mathrm{CO}_{2}$. IL-2 and IFN- $\gamma$ levels were determined on day 3 and day 5 , respectively. The cells were harvested on day 5 , and $\mathrm{CD} 4^{+} \mathrm{T}$ cell proliferation was measured by ${ }^{3} \mathrm{H}-\mathrm{TDR}$.

\section{Antibody-dependent cell-mediated cytotoxicity (ADCC) and} complement-dependent cytotoxicity (CDC) assays

For the ADCC assay, activated human $\mathrm{CD}^{+}{ }^{+} \mathrm{T}$ cells and serial dilutions of testing antibodies (from $66.7 \mathrm{nM}$ to $0.00667 \mathrm{pM}$ ) were preincubated in $96-$ well plates for $30 \mathrm{~min}$. PBMCs were then added at an effector/target ratio of 50:1. The plates were incubated at $37^{\circ} \mathrm{C}$ in a $5 \% \mathrm{CO}_{2}$ incubator for $4-6 \mathrm{~h}$. Target cell lysis was determined by an LDH-based cytotoxicity detection kit. The Herceptin-induced ADCC effect on SK-BR-3 cells was used as a positive control.

For the CDC assay, activated human $\mathrm{CD}^{+}{ }^{+} \mathrm{T}$ cells and serial dilutions of testing antibodies (from $200 \mathrm{nM}$ to $0.781 \mathrm{nM}$ ) were mixed in 96-well plates. Human complement was added at a dilution ratio of $1: 50$. The plates were incubated at $37^{\circ} \mathrm{C}$ in a
$5 \% \mathrm{CO}_{2}$ incubator for 2-3 h. Target cell lysis was determined by CellTiter-Glo. Rituxan ${ }^{\oplus}$-induced Ramos cell lysis was used as a positive control.

In vivo efficacy study in tumor models

All animals were maintained under specific pathogen-free conditions in the animal facilities of WuXi AppTec Co., Ltd. All animal-related experiments were approved by the Animal Use and Care Committee of WuXi AppTec Co., Ltd. MC38-hPD-L1 cells were subcutaneously (s.c.) implanted into the right flank of hPD-1 knock-in female mice (Nanjing Galaxy Biopharmaceutical Co., Ltd.). Mice were randomized with mean tumor volumes calculated at $\sim 74 \mathrm{~mm}^{3}$. Unmodified MC38 cells were s.c. implanted into the right flank of female C57BL/6 J mice (Shanghai SLAC Co., Ltd.). Mice were randomized with mean tumor volumes of $\sim 60 \mathrm{~mm}^{3}$. Mice were dosed intraperitoneally with isotype control or test antibody once every 3 days. Tumor growth inhibition (TGI) was calculated for each group using the following formula: TGI $(\%)=\left[1-\left(T_{\mathrm{i}}-T_{0}\right) /\left(V_{\mathrm{i}}-V_{0}\right)\right] \times 100 \quad\left(T_{\mathrm{i}}\right.$ and $V_{\mathrm{i}}$ : the average tumor volume of the treatment group and the isotype control group, respectively, on a given day; $T_{0}$ and $V_{0}$ : the average tumor volume of the treatment group and the isotype control group, respectively, on day 0 ).

PK study in cynomolgus monkeys

A single-dose PK study of CS1003 was conducted in cynomolgus monkeys at doses of 2,6 , and $18 \mathrm{mg} / \mathrm{kg}$. Nine female and nine male cynomolgus monkeys were assigned into three treatment groups and received one intravenous infusion of CS1003 on day 0. The repeat-dose group containing six animals received four doses of CS1003 at $6 \mathrm{mg} / \mathrm{kg}$ over 4 weeks (once per week). Blood samples were collected from each animal at predose $(0), 0.25 \mathrm{~h}$, $0.5 \mathrm{~h}, 1 \mathrm{~h}, 4 \mathrm{~h}, 8 \mathrm{~h}$, day 2 ( $24 \mathrm{~h})$, day 4 (72 h), day 7 (144h), day 14 $(312 \mathrm{~h})$, day $21(480 \mathrm{~h})$, day 28 (648 h), and day 35 (816 h) after the last dose and were processed for serum isolation. Serum concentrations of $\mathrm{CS} 1003$ were determined by the validated ELISA method with a dynamic range from 100 to $8000 \mathrm{ng} / \mathrm{mL}$. Anti-drug antibody (ADA) levels were also measured with bridging ELISA.

\section{PD-1 receptor occupancy}

For PD-1 receptor occupancy, CS1003 binding to PD-1 molecules on PBMCs was assessed by flow cytometry on circulating $\mathrm{CD}^{+} \mathrm{CD}^{+}{ }^{+} \mathrm{T}$ cells and $\mathrm{CD}^{+}{ }^{+} \mathrm{CD}^{+}{ }^{+} \mathrm{T}$ cells. Blood samples from the above monkey PK study were collected at predose (day -1$), 2$ h, 8 h, day 2 ( 24 h), day 3 (48 h), day 4 (72 h), day 7 (144 h), day 14 $(312 \mathrm{~h})$, and day $28(648 \mathrm{~h})$ after the single dose. Blood samples were preincubated $\left(60 \mathrm{~min}\right.$ at $\left.4{ }^{\circ} \mathrm{C}\right)$ with a saturating concentration of either unlabeled hlgG4 (isotype control) or CS1003, washed extensively, and then costained with $\mathrm{PE}$-conjugated antihuman IgG4, FITC-conjugated antihuman CD45, APC-conjugated antihuman CD3, PerCP-Cy5-conjugated antihuman CD4, and APC-Cy7conjugated antihuman CD8 antibodies for $30 \mathrm{~min}$ at $4{ }^{\circ} \mathrm{C}$ in the dark. The erythrocytes were lysed in $2 \mathrm{~mL} 1 \times$ BD FACS Lysing Solution for $15 \mathrm{~min}$ at room temperature. PBMCs were washed twice and analyzed by a Becton-Dickinson FACS Canto II flow cytometer. The receptor occupancy rate (\%RO) was calculated as follows: $\% \mathrm{RO}=(\mathrm{CS} 1003-\mathrm{PE}$ positive percent without CS1003 staining tube)/(CS1003-PE positive percent with CS1003 staining tube) $\times 100 \%$.

Statistical analysis

In vitro pharmacodynamic experiments, including $T$ cell proliferation response, IFN- $\gamma$ and IL-2 secretion results were analyzed by Wilcoxon matched pairs $t$ test with Graphpad Prism5. In vivo efficacy results were analyzed by independent sample $t$ test using SPSS 17.0. Differences with $P<0.05$ were considered significant. 


\section{RESULTS}

CS1003 selectively bound to PD-1 and blocked PD-1 interaction with its ligands

A panel of rat anti-PD-1 mAbs was generated through hybridoma technology. mAbs with favorable characteristics were further humanized. CS1003, a humanized, hinge stabilized IgG4 mAb, was selected as the lead candidate for further characterization. CS1003 potently bound to human, monkey and mouse PD-1 with $K_{\mathrm{D}}$ values of $6.13,8.74$, and $3.99 \mathrm{nM}$, respectively (Table 1). The binding of CS1003 to PD-1 proteins in three species was evaluated by ELISA and flow cytometry. As shown in Fig. 1 a and Supplementary Fig. S1a, CS1003 bound to human, mouse and cynomolgus monkey recombinant PD-1 with $\mathrm{EC}_{50}$ values of $0.1757,0.3664$, and 0.2459 $\mathrm{nM}$, respectively. $\mathrm{CS} 1003$ bound to cells expressing human, cynomolgus monkey and mouse PD-1 with $\mathrm{EC}_{50}$ values of 2.20, 1.47 , and $12.9 \mathrm{nM}$, respectively (Fig. 1b, Supplementary Fig. S1b, c). CS1003 did not bind to parental CHO-S cells (Supplementary Table S1). In addition, CS1003 was tested for its capability of interrupting PD-1/PD-L1 and PD-1/PD-L2 interactions. CS1003 competently inhibited the binding of human PD-1-PD-L1 and PDL2 (Fig. 1C, Supplementary Fig. S1d), mouse PD-1-PD-L1 (Supplementary Fig. S1e) and cynomolgus monkey PD-1-PD-L1

Table 1. Affinity of PD-1 antibody to hPD-1, cynoPD-1, and mPD-1 measured by SPR.

\begin{tabular}{lllll}
\hline Antigen & Ligand & ka $(1 / \mathrm{Ms})$ & kd $(1 / \mathrm{s})$ & $K_{\mathrm{D}}(\mathrm{M})$ \\
\hline hPD-1 & $\mathrm{CS} 1003$ & $5.97 \times 10^{5}$ & $3.66 \times 10^{-3}$ & $6.13 \times 10^{-9}$ \\
cynoPD-1 & $\mathrm{CS} 1003$ & $8.09 \times 10^{5}$ & $7.07 \times 10^{-3}$ & $8.74 \times 10^{-9}$ \\
mPD-1 & $\mathrm{CS} 1003$ & $3.23 \times 10^{5}$ & $1.29 \times 10^{-3}$ & $3.99 \times 10^{-9}$ \\
hPD-1 & Pembrolizumab & $8.79 \times 10^{5}$ & $2.28 \times 10^{-3}$ & $2.59 \times 10^{-9}$ \\
\hline
\end{tabular}
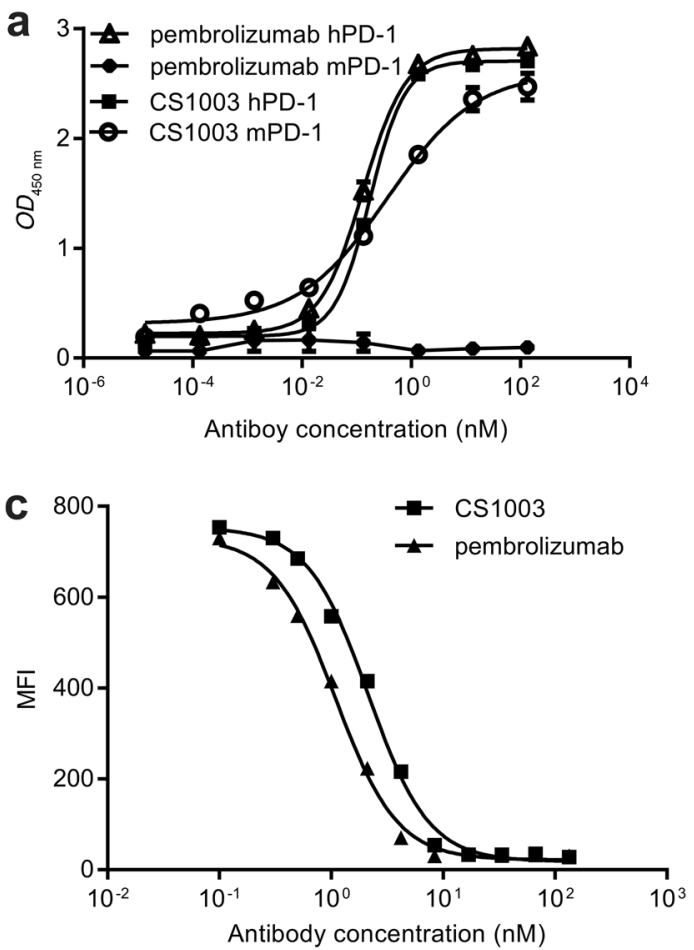

(Supplementary Fig. S1f). The binding potency of CS1003 to human and monkey PD-1 was comparable to that of pembrolizumab; moreover, CS1003 cross-reacts with mouse PD-1. Similar to pembrolizumab, CS1003 exhibited the ability to bind human PD-1 but not other immunoglobulin superfamily proteins (CD28, CTLA-4, ICOS, and BTLA) (Fig. 1d). These results indicate that CS1003 potently binds to human, cynomolgus monkey and mouse PD-1 and efficiently blocks the PD-1/PD-L1 and PD-1/PD-L2 interactions.

CS1003 enhanced T cell proliferation and cytokine secretion Blocking the PD-1 interaction with its ligand results in enhanced T cell proliferation and cytokine secretion [10]. Therefore, CS1003 activity in regulating $T$ cell function was measured using the MLR assay. CS1003 significantly increased IL-2 and IFN- $\gamma$ levels in T cells in a dose-dependent manner similar to that of pembrolizumab (Fig. 2a, b). Using the $\left[{ }^{3} \mathrm{H}\right]$-TDR system, $\mathrm{CS} 1003$ was shown to induce dosedependent $T$ cell proliferation with comparable efficacy to pembrolizumab (Fig. 2c). To assess whether CS1003 is capable of inducing nonspecific cytokine release in a TCR activation-dependent manner, the ability of CS1003 to induce T cell quiescence was determined. Freshly isolated human PBMCs (five donors) were incubated with 66.7 or $667 \mathrm{nM} \mathrm{CS1003.} \mathrm{The} \mathrm{results} \mathrm{showed} \mathrm{that}$ CS1003 had no significant influence on nonspecific cytokine release (data not shown). In summary, CS1003 promotes T cell proliferation and cytokine secretion in vitro.

CS1003 inhibited Treg cell suppressive function

PD-1-expressing Treg cells modulate $T$ cell function in the tumor microenvironment [11]. Hence, the role of $\mathrm{CS} 1003$ in restoring $T$ cell function suppressed by Treg cells was explored. CD $4^{+} \mathrm{CD} 25^{-}$ effector $T$ (Teff) cell proliferation and cytokine secretion were suppressed by allogeneic Treg cells. As shown in Fig. 2d, e, CS1003 completely restored $\mathrm{CD}^{+}{ }^{+} \mathrm{CD} 25^{-} \mathrm{T}$ cell cytokine production and proliferation. Taken together, the results show that CS1003

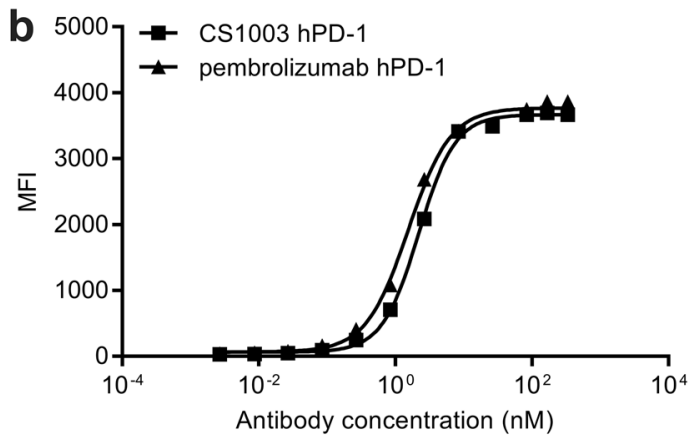

d

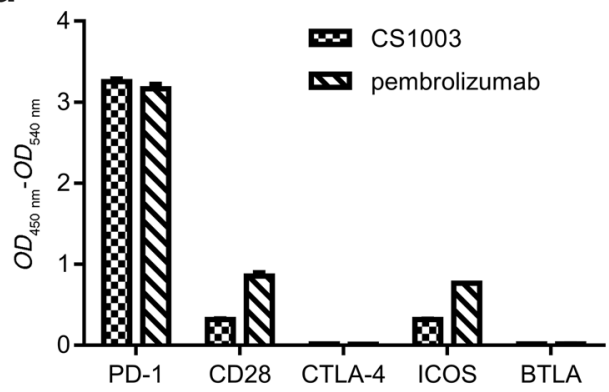

Fig. 1 PD-1 antibodies bind to the PD-1 protein and block the PD-1/PD-L1 and PD-1/PD-L2 interactions. a Binding of CS1003 or pembrolizumab to plate-coated recombinant human and mouse PD-1 proteins was assessed by ELISA. b Binding of CS1003 or pembrolizumab to cell surface human PD-1 was determined by FACS. $c$ The functionality of CS1003 or pembrolizumab in blocking human PD1/PD-L1 interactions was assessed by flow cytometry. d Binding of CS1003 or pembrolizumab to human PD-1, CD28, CTLA-4, ICOS, or BTLA was assessed by ELISA. 



Fig. 2 PD-1 blockade enhances T cell function. a-c The functionality of CS1003 and pembrolizumab in enhancing T cell responses was assessed using MLR, and T cell proliferation ( $\left[{ }^{3} \mathrm{H}\right]-\mathrm{TDR}$ incorporation) and effector function (IFN- $\gamma$ production) were quantified. $\mathbf{d}-\mathbf{e}$ CS1003 reversed Treg cell suppressive function in the MLR assay. ${ }^{* *} P<0.01,{ }^{* * *} P<0.001$.
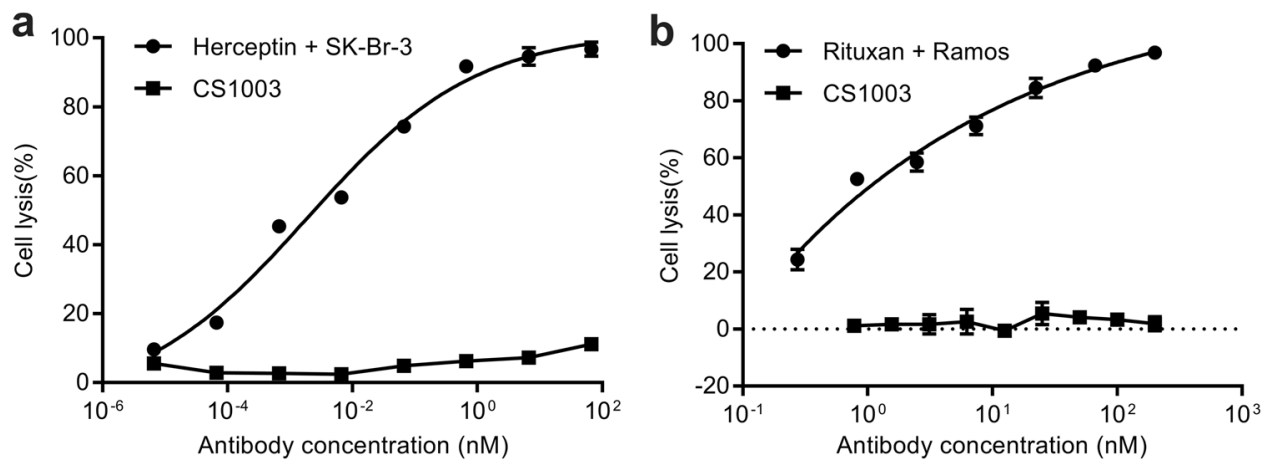

Fig. 3 CS1003 showed no ADCC or CDC activity. The ADCC (a) and CDC (b) activities of CS1003 and pembrolizumab were evaluated. Herceptin-induced SK-Br-3 cell lysis and rituximab-induced Ramos cell lysis were used as positive controls for ADCC and CDC, respectively. CPM, counts per minute.

abrogates Treg cell suppression of T cell proliferation and cytokine production.

CS1003 showed no ADCC or CDC activity

The lgG4 isotype is known to have a low effector function, which makes it ideal as a therapeutic blocking antibody [12]. CS1003 is a humanized IgG4 mAb. ADCC activities induced by CS1003 were assessed using human PBMCs as effector cells and activated human $\mathrm{CD4}^{+} \mathrm{T}$ cells as target cells. As demonstrated in Fig. 3a, CS1003 did not induce ADCC activities on activated human $\mathrm{CD}^{+}{ }^{+} \mathrm{T}$ cells, avoiding potential damage to $\mathrm{PD}$-1-positive lymphocytes. The CDC activities induced by CS1003 on activated 
human $\mathrm{CD}^{+}{ }^{+} \mathrm{T}$ cells were further evaluated. As shown in Fig. 3b, compared with the positive control rituximab, CS1003 had no apparent $\mathrm{CDC}$ activity within the concentration range of 0.781-200 nM. The ADCC and CDC activities of CS1003 were comparable to those of pembrolizumab (Supplementary Fig. S2). In summary, no ADCC or CDC activity was observed using CS1003.

\section{CS1003 significantly inhibited MC38-hPD-L1 and MC38 tumor} growth

The in vivo antitumor efficacy of CS1003 was evaluated using the MC38-hPD-L1 colon cancer model in hPD-1 knock-in mice. Mean tumor volumes were calculated on day 21 post-treatment (Fig. 4a), and the tumor images are shown in Fig. 4b. Compared with the isotype control, CS1003 exhibited a significant tumor growth inhibition (TGI) of $82.0 \%, 71.1 \%$ and $101.7 \%$ at $0.1,0.5$, and $2.5 \mathrm{mg} /$ $\mathrm{kg}$, respectively. In this study, nivolumab showed a TGI of $89.3 \%$ at $2.5 \mathrm{mg} / \mathrm{kg}$, suggesting that the antitumor effect of CS1003 was
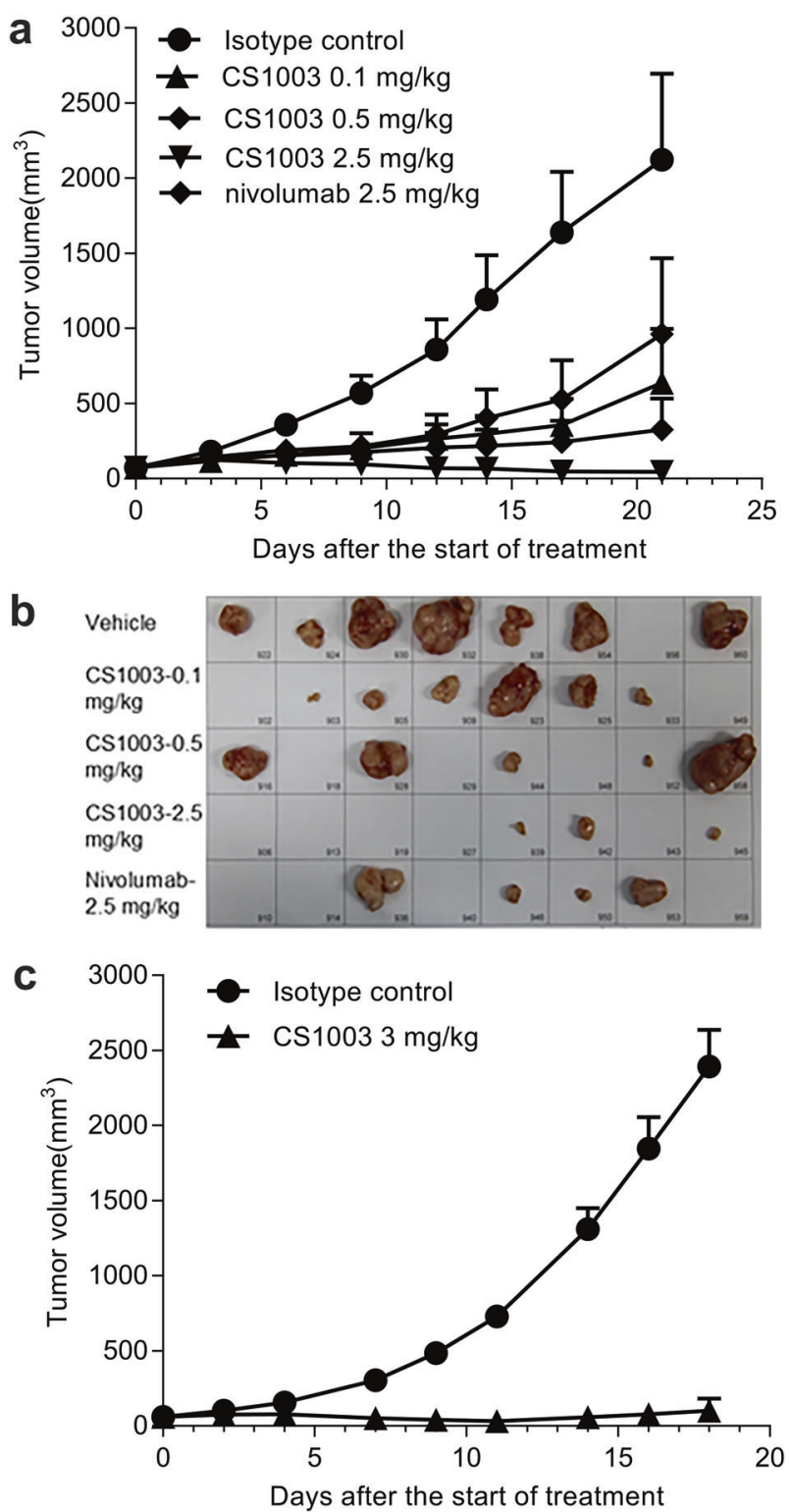

Fig. 4 The in vivo efficacy of $\mathrm{CS} 1003$ was evaluated in an established MC38-hPD-L1 model (a and b) and a parental MC38 syngeneic model (c). All antibodies were administered intraperitoneally (i.p.) every 3 days (Q3D). Mean \pm SEM, $n=8$. comparable to that of nivolumab. No abnormal body weight changes or signs of toxicity were observed throughout the study. CS1003 also demonstrated a significant TGI effect with a TGI of $98.2 \%$ at $3 \mathrm{mg} / \mathrm{kg}$ in the unmodified MC38 model (Fig. 4c). Hence, CS1003 showed strong in vivo efficacy in both the parental MC38 model and the MC38-hPD-L1 tumor model.

CS1003 showed typical PK profiling and high receptor occupancy A pharmacokinetics study of $\mathrm{CS} 1003$ was conducted in cynomolgus monkeys following a single intravenous infusion at doses of 2, 6 , and $18 \mathrm{mg} / \mathrm{kg}$. As shown in Fig. $5 \mathrm{a}$ and Table 2, the average initial concentration $\left(C_{0}\right)$ values were $58.9,178$, and $492 \mu \mathrm{g} / \mathrm{mL}$ and the $\mathrm{AUC}_{0 \text {-last }}$ values were 331,1070 , and $4580 \mu \mathrm{g} \cdot \mathrm{day} / \mathrm{mL}$ for animals in the 2,6 , and $18 \mathrm{mg} / \mathrm{kg}$ dose groups, respectively. Thus, the systemic exposure of CS1003 increased dose proportionally within the dose range from 2 to $18 \mathrm{mg} / \mathrm{kg}$. The average serum clearance $(\mathrm{CL})$ values were $6.80,6.55$, and $3.98 \mathrm{~mL} \cdot \mathrm{d}^{-1} \cdot \mathrm{kg}^{-1}$ and the elimination half-life $\left(T_{1 / 2}\right)$ values were $6.36,5.67$, and 11.5 days in the 2,6 , and $18 \mathrm{mg} / \mathrm{kg}$ dose groups, respectively. The volume of distribution at steady-state $\left(V d_{\mathrm{ss}}\right)$ was similar across groups, in the range from 0.0514 to $0.0631 \mathrm{~L} / \mathrm{kg}$. No obvious sex difference was observed in systemic exposure and the calculated PK parameters at the tested doses.

In the repeated dose PK study, following the 4th iv administration of CS1003 at $6 \mathrm{mg} / \mathrm{kg}$, the average $C_{0}$ and $A U C_{\text {last }}$ values were comparable with those of the single administration, suggesting no marked accumulation after the 4th dosing in either sex.

ADAs were detected from $312 \mathrm{~h}$ postdose in the 2 and $6 \mathrm{mg} / \mathrm{kg}$ single-dose groups and from $648 \mathrm{~h}$ postdose in the $18 \mathrm{mg} / \mathrm{kg}$ group. The ADA positive rate was $36.7 \%$ at $2 \mathrm{mg} / \mathrm{kg}, 73.3 \%$ at 6 $\mathrm{mg} / \mathrm{kg}$ and $10.0 \%$ at $18 \mathrm{mg} / \mathrm{kg}$. In the repeated dose group, the positive rate was $30 \%$. The decrease in the ADA positive rate in the high-dose group might be due to drug tolerance when the drug concentration is high. In the repeated dose group, the ADA positive rate was $30 \%$ after the 4 th dose of CS1003. Overall, the immunogenicity of CS1003 in cynomolgus monkeys was lower than that reported in previous data for pembrolizumab and nivolumab.

Receptor occupancy is an important index for anti-PD-1 mAb efficacy evaluation [13]. As shown in Fig. 5b, c, the receptor occupancy rate quickly increased following single iv administration of CS1003 at 2, 6, and $18 \mathrm{mg} / \mathrm{kg}$ and was saturated at $24 \mathrm{~h}$ postdose in all groups until day 14 (312 h). Decreased receptor occupancy was noted in most animals in the $2 \mathrm{mg} / \mathrm{kg}$ and $6 \mathrm{mg} / \mathrm{kg}$ dose groups on day 28 , but the averaged receptor occupancy rate remained above $50 \%$. However, in the $18 \mathrm{mg} / \mathrm{kg}$ dose group, receptor occupancy was still saturated on day 28 .

\section{DISCUSSION}

The application of immune checkpoint inhibitors has changed the paradigm in cancer treatment. Anti-PD-1 antibodies, including nivolumab, pembrolizumab, cemiplimab, JS-001, sintilimab, and camrelizumab, have been approved or are being evaluated for the treatment of more than ten cancer types, such as metastatic melanoma, non-small-cell lung cancer, head and neck cancer, Hodgkin's lymphoma, urothelial carcinoma, gastric cancer, cervical cancer, hepatocellular carcinoma, Merkel cell carcinoma, renal cell carcinoma, small-cell lung cancer, esophageal carcinoma, and colorectal cancer.

CS1003 is a humanized lgG4 PD-1 mAb developed by CStone Pharmaceuticals (Suzhou) Co., Ltd. This study included the in vitro and in vivo characterization as well as a PK evaluation of CS1003. Either pembrolizumab or nivolumab was used as a reference in this study, and the results were equivalent to those previously reported. CS1003 binds to human, cynomolgus monkey and mouse PD-1 with high affinity and blocks the interaction of PD-1 

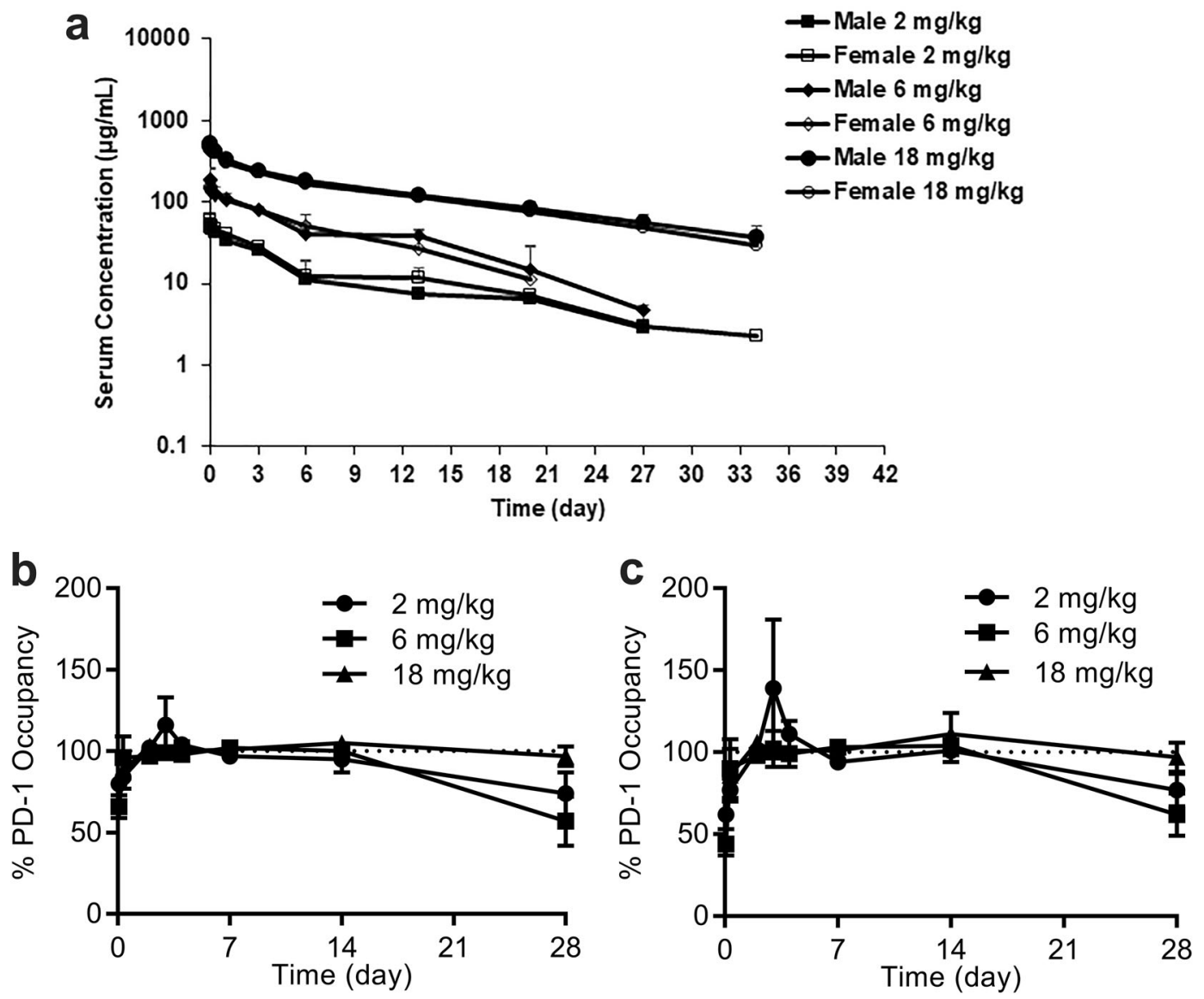

Fig. 5 a Concentration-time profile (mean \pm SD) of CS1003 after a single dose was administered to naive cynomolgus monkeys ( 3 males +3 females per dose group). $\mathbf{b}$ The percentages of occupied PD-1 receptors on total T cells (b) and CD8 ${ }^{+}$cells (c) were determined by flow cytometry.

Table 2. Pharmacokinetic parameters following single i.v. injection of CS1003 in cynomolgus monkey (mean \pm SD, $n=6$ ).

\begin{tabular}{lccc}
\hline Group & $2 \mathrm{mg} / \mathrm{kg}$, i.v. & $6 \mathrm{mg} / \mathrm{kg}$, i.v. & $18 \mathrm{mg} / \mathrm{kg}$, i.v. \\
\hline$C_{0}(\mu \mathrm{g} / \mathrm{mL})$ & $58.9 \pm 9.18$ & $178 \pm 47.5$ & $492 \pm 40.5$ \\
$T_{1 / 2}(\mathrm{~d})$ & $6.36 \pm 2.83$ & $5.67 \pm 6.05$ & $11.5 \pm 3.25$ \\
$V d_{\mathrm{ss}}(\mathrm{L} / \mathrm{kg})$ & $0.0577 \pm 0.0134$ & $0.0514 \pm 0.0124$ & $0.0631 \pm 0.00704$ \\
$\mathrm{Cl}\left(\mathrm{mL} \cdot \mathrm{d}^{-1} \cdot \mathrm{k}^{-1}\right)$ & $6.80 \pm 3.12$ & $6.55 \pm 2.50$ & $3.98 \pm 0.488$ \\
$\mathrm{AUC}_{\text {o-last }}(\mu \mathrm{g} \cdot \mathrm{d} / \mathrm{mL})$ & $310 \pm 101$ & $978 \pm 358$ & $3980 \pm 323$ \\
$\mathrm{AUC}_{\text {0-inf }}(\mu \mathrm{g} \cdot \mathrm{d} / \mathrm{mL})$ & $331 \pm 99.3$ & $1070 \pm 507$ & $4580 \pm 626$ \\
& & & \\
\end{tabular}

with both PD-L1 and PD-L2 ligands. As reported, human PD-L1 also cross-reacts with mouse PD-1 [14]. R9 is a rat hybridoma of CS1003 that interacts with PD-1 in similar manner to human PD-L1 [15]. The cross-reactivity to both human and mouse PD-1 makes $\mathrm{CS} 1003$ to be the most straightforward and cost-effective approach for testing in mouse models. CS1003 resulted in significant TGI in both MC38-hPD-L1 colon cancer models in human PD-1 knock-in mice with comparable potency to nivolumab. Furthermore, CS1003 also showed antitumor activity in syngeneic tumor models, i.e., CT26 and MC38 models, without additional modification. These results clearly demonstrated its equivalent ability to target both human and mouse PD-1 and enhance antitumor immunity.
Although the clinical success rate of anti-PD- 1 mAbs has been demonstrated to be substantially higher than that of other cancer therapies, $30 \%-60 \%$ of patients show no response to PD-1 blockade and are refractory to PD-1 treatment [8]. Combination therapy has become an important strategy to increase the response rate and overcome resistance. Preclinical evidence is essential to provide rationale for agent/target selection in combination strategies. Among the approved anti-PD-1 antibodies, none of them cross-reacted with mouse. Surrogate antibodies, such as RMP-1-14 from Bio X Cell, are generated and used to evaluate the efficacy in syngeneic animal models. However, due to the difference in binding epitopes, surrogate antibodies may not fully reflect the actual function and potential biology of therapeutic antibodies in humans. The cross-reaction of CS1003 with human and mouse PD-1 provides a significant advantage to test the efficacy of its combination with a variety of other antitumor agents in syngeneic mouse tumor models, which is the focus of ongoing efforts at our institution. The potential of CS1003 combined with chemotherapy or targeted therapy is now under evaluation in syngeneic models. CS1003 shows promising effects when combined with CStone's MEK inhibitor (CS3006) and CDK4/6 inhibitor (CS3002) in the CT26 model (data not shown). Other potential combinations with new molecules from global partners are also under investigation.

CS1003 showed a linear PK profile within the dose range of 2-18 $\mathrm{mg} / \mathrm{kg}$ following single intravenous administration in monkeys. The PK parameters, including $\mathrm{CL}$ and terminal half-life, were within the typical ranges seen for lgGs and were comparable to those of pembrolizumab and nivolumab $[16,17]$. The ADA incidence was 
low, suggesting that $\mathrm{CS} 1003$ induces low immunogenicity in monkeys.

The mechanism of action of anti-PD-1 antibodies includes a complex interplay of receptor binding, target engagement and positive feedback mechanisms [18]. Receptor occupancy is an important pharmacodynamic marker for therapeutic antibody evaluation. Since nivolumab has been extensively studied in clinical trials $[13,19]$, we optimized the receptor occupancy assay of nivolumab to examine the PD-1 occupancy of CS1003 on circulating $\mathrm{CD}^{+} \mathrm{T}$ cells, including $\mathrm{CD}^{+} \mathrm{CD}^{+} \mathrm{T}$ cells and $\mathrm{CD}^{+} \mathrm{CD}^{+} \mathrm{T}$ cells. The $\mathrm{RO} \%$ of $\mathrm{CS} 1003$ quickly reached $\sim 100 \%$ from $24 \mathrm{~h}$ postdose in all single-dose groups. Compared with the PD-1 occupancy of nivolumab, which appeared to be doseindependent, with a mean peak occupancy of $85 \%$ observed at 4-24 $\mathrm{h}$ after one infusion in patients, the consistently high levels of PD-1 occupancy observed after CS1003 treatment indicate its potentially better efficacy.

In conclusion, CS1003 is a humanized IgG4 anti-PD-1 mAb with mouse cross-reactivity. The preclinical data are promising and provide the basis for its efficacy and safety evaluation in clinical trials.

\section{ACKNOWLEDGEMENTS}

We thank Dr. Yan-fen Teng, Quan Qiu, and Ru-mei Chen for language assistance during the preparation of this manuscript.

\section{AUTHOR CONTRIBUTIONS}

$\mathrm{FL}, \mathrm{JL}, \mathrm{KY}, \mathrm{JZ}, \mathrm{ZHL}, \mathrm{LL}, \mathrm{YWB}, \mathrm{ZQ}, \mathrm{YZ}, \mathrm{BTY}$, and JL designed and performed the research; $\mathrm{FL}, \mathrm{ZHL}, \mathrm{LL}, \mathrm{YWB}, \mathrm{ZQ}$, and $\mathrm{XW}$ wrote the paper; $\mathrm{XW}$ approved the version to be published.

\section{ADDITIONAL INFORMATION}

The online version of this article (https://doi.org/10.1038/s41401-020-0422-6) contains supplementary material, which is available to authorized users.

Competing interests: The authors declare no competing interests.

\section{REFERENCES}

1. Marin-Acevedo JA, Dholaria B, Soyano AE, Knutson KL, Chumsri S, Lou Y. Next generation of immune checkpoint therapy in cancer: new developments and challenges. J Hematol Oncol. 2018;11:39.

2. Fife BT, Pauken KE. The role of the PD-1 pathway in autoimmunity and peripheral tolerance. Ann NY Acad Sci. 2011;1217:45-59.
3. Francisco LM, Sage PT, Sharpe AH. The PD-1 pathway in tolerance and autoimmunity. Immunol Rev. 2010;236:219-42.

4. Bardhan K, Anagnostou T, Boussiotis VA. The PD1:PD-L1/2 pathway from discovery to clinical implementation. Front Immunol. 2016;7:550.

5. Ishida Y, Agata Y, Shibahara K, Honjo T. Induced expression of PD-1, a novel member of the immunoglobulin gene superfamily, upon programmed cell death. EMBO J. 1992;11:3887-95.

6. Topalian SL, Sznol M, McDermott DF, Kluger HM, Carvajal RD, Sharfman WH, et al. Survival, durable tumor remission, and long-term safety in patients with advanced melanoma receiving nivolumab. J Clin Oncol. 2014;32:1020-30.

7. Fuchs CS, Doi T, Jang RW, Muro K, Satoh T, Machado M, et al. Safety and efficacy of pembrolizumab monotherapy in patients with previously treated advanced gastric and gastroesophageal junction cancer: phase 2 clinical KEYNOTE-059 trial. JAMA Oncol. 2018;4:e180013.

8. Song $M$, Chen $X$, Wang $L$, Zhang $Y$. Future of anti-PD-1/PD-L1 applications: combinations with other therapeutic regimens. Chin J Cancer Res. 2018;30:157-72.

9. Chowdhury PS, Chamoto K, Honjo T. Combination therapy strategies for improving PD-1 blockade efficacy: a new era in cancer immunotherapy. J Intern Med. 2018;283:110-20.

10. Brown JA, Dorfman DM, Ma FR, Sullivan EL, Munoz O, Wood CR, et al. Blockade of programmed death-1 ligands on dendritic cells enhances $T$ cell activation and cytokine production. J Immunol. 2003;170:1257-66.

11. Wang W, Lau R, Yu D, Zhu W, Korman A, Weber J. PD1 blockade reverses the suppression of melanoma antigen-specific $\mathrm{CTL}$ by $\mathrm{CD}^{+} \mathrm{CD} 25(\mathrm{Hi})$ regulatory T cells. Int Immunol. 2009;21:1065-77.

12. Chen DS, Irving BA, Hodi FS. Molecular pathways: next-generation immunotherapy-inhibiting programmed death-ligand 1 and programmed death-1. Clin Cancer Res. 2012;18:6580-7.

13. Topalian SL, Hodi FS, Brahmer JR, Gettinger SN, Smith DC, McDermott DF, et al. Safety, activity, and immune correlates of anti-PD-1 antibody in cancer. N Engl J Med. 2012;366:2443-54.

14. Huang A, Peng D, Guo H, Ben Y, Zuo X, Wu F, et al. A human programmed deathligand 1-expressing mouse tumor model for evaluating the therapeutic efficacy of anti-human PD-L1 antibodies. Sci Rep. 2017;7:42687.

15. Li D, Xu J, Wang Z, Gong Z, Liu J, Zheng Y, et al. Epitope mapping reveals the binding mechanism of a functional antibody cross-reactive to both human and murine programmed death 1. MAbs. 2017;9:628-37.

16. Merck \& Co. I. Pharmacology Review of Keytruda (pembrolizumab). U.S. Food and Drug Administration website. August 22, 2014 https://www.accessdata.fda.gov/ drugsatfda_docs/nda/2014/1255140rig1s000PharmR.pdf.

17. Wang $C$, Thudium KB, Han M, Wang XT, Huang $H$, Feingersh D. et al. In vitro characterization of the anti-PD-1 antibody nivolumab, BMS-936558, and in vivo toxicology in non-human primates. Cancer Immunol Res. 2014;2:846-56.

18. Okazaki T, Chikuma S, Iwai $Y$, Fagarasan S, Honjo T. A rheostat for immune responses: the unique properties of PD-1 and their advantages for clinical application. Nat Immunol. 2013;14:1212-8.

19. Brahmer JR, Drake CG, Wollner I, Powderly JD, Picus J, Sharfman WH, et al. Phase I study of single-agent anti-programmed death-1 (MDX-1106) in refractory solid tumors: safety, clinical activity, pharmacodynamics, and immunologic correlates. J Clin Oncol. 2010;28:3167-75. 